\title{
On the relationship between auroral absorption, electrojet currents and plasma convection
}

\author{
A. C. Kellerman ${ }^{1}$, R. A. Makarevich ${ }^{1}$, F. Honary ${ }^{2}$, and T. L. Hansen ${ }^{3}$ \\ ${ }^{1}$ Department of Physics, La Trobe University, Victoria, 3086, Australia \\ ${ }^{2}$ Department of Communication Systems, Lancaster University, Lancaster, LA1 4WA, UK \\ ${ }^{3}$ Troms $\varnothing$ Geophysical Observatory, University of Troms $\varnothing$, Troms $\varnothing, 9037$, Norway
}

Received: 3 June 2008 - Revised: 25 November 2008 - Accepted: 10 December 2008 - Published: 2 February 2009

\begin{abstract}
In this study, the relationship between auroral absorption, electrojet currents, and ionospheric plasma convection velocity is investigated using a series of new methods where temporal correlations are calculated and analysed for different events and MLT sectors. We employ cosmic noise absorption (CNA) observations obtained by the Imaging Riometer for Ionospheric Studies (IRIS) system in Kilpisjärvi, Finland, plasma convection measurements by the European Incoherent Scatter (EISCAT) radar, and estimates of the electrojet currents derived from the Troms $\varnothing$ magnetometer data. The IRIS absorption and EISCAT plasma convection measurements are used as a proxy for the particle precipitation component of the Hall conductance and ionospheric electric field, respectively. It is shown that the electrojet currents are affected by both enhanced conductance and electric field but with the relative importance of these two factors varying with magnetic local time (MLT). The correlation between the current and electric field (absorption) is the highest at 12:0015:00 MLT (00:00-03:00 MLT). It is demonstrated that the electric-field-dominant region is asymmetric with respect to magnetic-noon-midnight meridian extending from 09:00 to 21:00 MLT. This may be related to the recently reported absence of mirror-symmetry between the effects of positive and negative IMF $B_{y}$ on the high-latitude plasma convection pattern. The conductivity-dominant region is somewhat wider than previously thought extending from 21:00 to 09:00 MLT with correlation slowly declining from midnight towards the morning, which is interpreted as being in part due to highenergy electron clouds gradually depleting and drifting from midnight towards the morning sector. The conductivitydominant region is further investigated using the extensive
\end{abstract}

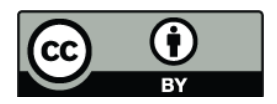

Correspondence to: R. A. Makarevich (r.makarevich@latrobe.edu.au)
IRIS riometer and Troms $\varnothing$ magnetometer datasets with results showing a distinct seasonal dependence. The region of high current-absorption correlation extends from 21:00 to 06:00 MLT near both equinoxes, however, it is narrower and rotated towards the morning (02:00-07:00 MLT) in summer, while in winter the correlation shows much greater variability with MLT. During periods of high current-electricfield correlation, the relationship between electric field and absorption can be described as an inverse proportionality, which can be explained by limitation of the electrojet current by the magnetospheric generator. Possible cases of electron heating absorption are also investigated with absorption showing no obvious dependence on the ion velocity or electron temperature.

Keywords. Ionosphere (Auroral ionosphere; Electric fields and currents; Particle precipitation)

\section{Introduction}

Energetic particle precipitation in the auroral ionosphere with its spectacular auroral displays represents arguably the most impressive aspect of Space Weather. Magnetospheric particles in the $0.5-20 \mathrm{keV}$ energy range precipitating in the ionospheric E- and F-regions are mostly responsible for the visual aurora, extensively studied with ground- and satellite-based optical instruments. More energetic particles $(>20 \mathrm{keV})$ enhance electron densities in the lower E- and Dregions $(<100 \mathrm{~km})$. The higher abundance of free electrons absorb more extra-terrestrial (ET) radio waves in the lower VHF frequency range $(30-50 \mathrm{MHz})$ resulting in less ET radio wave intensity reaching the Earth's surface. This phenomenon is known as cosmic noise absorption or CNA. It is routinely measured by ground-based relative ionospheric

Published by Copernicus Publications on behalf of the European Geosciences Union. 
opacity meters (riometers), which are widely used to monitor and study high-energy particles and their precipitation (see, for example, reviews by Stauning, 1996a,b).

The enhanced electron densities in the lower ionosphere also result in enhanced electrical conductances (heightintegrated conductivities) and, for constant electric fields, intensification of the auroral electrojet current. The changes in the electrojet currents are monitored by magnetometers that measure perturbations in the local geomagnetic field (e.g. Amm, 2001). The ambient plasma, consisting of cold particles with energies of $<1 \mathrm{keV}$ in the crossed electric $\boldsymbol{E}$ and magnetic $\boldsymbol{B}$ fields, move with the $\boldsymbol{E} \times \boldsymbol{B}$ convection drift. Much research effort has been devoted to studies of global ionospheric plasma convection, as this information is critical to our understanding of the magnetospheric-ionospheric system and the ionospheric response to changes or transitions from one IMF state to another (e.g. Ruohoniemi et al., 2002).

Understanding the relationships between various auroral phenomena has been at the heart of space physics research for the past 50 years (e.g. Meng et al., 1991). The magnetosphere and solar wind are coupled to the auroral ionosphere and hence studies of relationships between auroral phenomena are directly relevant to these coupling processes. Despite significant progress, there is still considerable scope to increase and refine the understanding of the links between plasma convection, conductances and current systems observed at high altitudes, as discussed below.

Auroral absorption and electrical conductances are closely related as both are height-integrated characteristics proportional to the plasma density and the electron collision frequency. This was confirmed experimentally by Walker and Bhatnagar (1989) and, more recently, by Senior et al. (2007). The Hall conductance $\Sigma_{H}$ was shown to be more strongly related to CNA than Pedersen conductance $\Sigma_{P}$ and the correlation between $\Sigma_{H}$ and CNA was shown to vary with magnetic local time or MLT (Senior et al., 2007). The correlation between $\Sigma_{H}$ and CNA was found to be considerable in all MLT sectors except for 15:00-19:00 MLT.

The CNA measured by a riometer has also been shown to depend indirectly on the electric field. This occurs when strong electric fields drive plasma waves that in turn interact with electrons increasing their temperatures and effective collision frequencies (St.-Maurice et al., 1981; Schlegel and St.-Maurice, 1981). The enhanced collision frequency may result in an increased CNA and such events were indeed identified in the polar cap, where the much stronger effects due to enhanced precipitation are not as pronounced as in the auroral zone (Stauning, 1984). These events are known as electron heating absorption or EHA events (Stauning, 1996a).

The electrojet current intensity has been shown to depend on both the electric field and the plasma density/conductivity, as expected from Ohm's law. Kamide and Vickrey (1983) demonstrated that the westward electrojet is split into two regions: a conductivity-dominant region and an electric-fielddominant region. The location of the transition within the westward electrojet has been found to vary with latitude, the higher the latitude, the earlier in MLT it occurs (see their Fig. 6). A similar latitude dependence has been found for the pre-midnight transition between the eastward and westward electrojets, coinciding with a transition from an electricfield- to a conductivity-dominant regime (Kamide and Vickrey, 1983; Davies and Lester, 1999). Kamide and Vickrey (1983) thus found that the electric field exerts a dominant role in the evening and late morning sectors. More recently, Sugino et al. (2002) showed that the conductivity-dominant region can extend to 08:00 MLT just after the dawn terminator.

Previous studies of the current-conductance-E-field relationship have mostly used the data collected using incoherent scatter radars (ISRs) that provide plasma convection velocity (electric field) data and information on conductivities in the limited range of altitudes (typically 90-300 km). From this information the ionospheric currents are inferred using Ohm's law (e.g. Davies and Lester, 1999; Sugino et al., 2002). In this approach all 3 parameters are provided by the same instrument at the same resolution and all the measurements are coincident and simultaneous. On the other hand, ISR is an expensive instrument with which to observe processes in the upper atmosphere. As a result they typically do not operate continuously for extended periods of time, i.e. months or years. Riometers and magnetometers, on the other hand, are passive instruments and are inexpensive to operate. As a result they function nearly continuously, under all conditions. They also provide observations integrated over the full ionospheric height profile. In addition, in previous studies, the solar and particle components of conductances were not considered separately as current depends on the total Hall and Pedersen conductances. Previous studies have also concentrated on nightside observations with the full MLT dependence including dayside sectors still less investigated.

In this study, we employ the CNA riometer measurements as a proxy for conductance in conjunction with the electric field and current measurements by an incoherent radar and magnetometer, respectively, to statistically investigate the relationships between the ionospheric electric fields, electrical conductances and current systems. A significant dataset is employed in order (1) to assess the potential of riometers in studies involving conductance estimates and in particular in studies of the relative importance of the electric field and conductance to ionospheric currents in all time sectors including the dayside and (2) to investigate the electric-fieldabsorption relationship and to determine whether electron heating absorption events are observed in the auroral zone.

\section{Experimental data}

In this study we used CNA measurements obtained by the Imaging Riometer for Ionospheric Studies (IRIS) in Kilpisjärvi, Finland $\left(69.1^{\circ} \mathrm{N}, 20.8^{\circ} \mathrm{E}, 65.90^{\circ} \mathrm{MLAT}\right)$. It 
operates at $38.2 \mathrm{MHz}$ and uses a single phased array of $8 \times 8$ dipole antennae to form 50 beams: one wide beam and 49 narrow beams as shown in Fig. 1 (Browne et al., 1995). The time resolution is $1 \mathrm{~s}$ although some post-integration is usually employed in order to increase the signal-to-noise ratio with a post-integration period of $1 \mathrm{~min}$ typically used. Similar to numerous previous studies, the absorption measurements in $\mathrm{dB}$ employed in this study were obtained from the IRIS raw power using methods and techniques implemented in the standard IRIS software (Marple and Honary, 2004).

The European Incoherent Scatter (EISCAT) tri-static UHF $(929.5 \mathrm{MHz})$ radar was also used in this study (Rishbeth and Williams, 1985). It consists of 3 parabolic dish antennas. The main antenna, located near Troms $\varnothing$ in Norway $\left(69.6^{\circ} \mathrm{N}, 19.2^{\circ} \mathrm{E}, 66.2^{\circ}\right.$ MLAT, Fig. 1$)$, combines both transmitting and receiving capabilities. The other two remote receivers are located in Sodankylä, Finland $\left(67.4^{\circ} \mathrm{N}, 26.6^{\circ} \mathrm{E}\right.$, 63.6 $6^{\circ}$ MLAT) and Kiruna, Sweden $\left(67.9^{\circ} \mathrm{N}, 20.5^{\circ} \mathrm{E}, 64.5^{\circ}\right.$ MLAT). In the CP1 common program, the data from which were used in this study, the UHF transmitter beam from Troms $\varnothing$ is aligned with the local F-region magnetic-field-line direction at an elevation of about $77^{\circ}$ and azimuth of $182^{\circ}$.

The EISCAT radar measures the ion and electron temperatures, plasma density and line-of-sight ion velocity. The full ion velocity vector is determined within the tri-static volume. In the relatively collisionless regime of the F-region, the ions move with the $\boldsymbol{E} \times \boldsymbol{B}$ velocity, and hence the ion velocity measurements by EISCAT are representative of the electric field (e.g. Davies et al., 1999; Davies and Lester, 1999). Figure 1 displays the location of the tri-static volume and the receiver beam directions (in blue) in CP1-K mode of EISCAT. The red (green) circle represents the location of the tri-static (projected tri-static) volume at 278-km (110-km) altitude. As the electric field is approximately the same along the magnetic field line, the tri-static volume was projected down to $110 \mathrm{~km}$ using the IGRF model. Figure 1 shows that in the Eand D-regions, it is the closest to the IRIS beam 16 and in this study the data from this beam were employed.

The International Monitor for Auroral Geomagnetic Effects (IMAGE) Troms $\varnothing$ magnetometer measures local magnetic perturbations in three perpendicular directions (Lühr et al., 1998). The position of the magnetometer is indicated by a red triangle in Fig. 1, which maps close to the projected tri-static volume. The 20 -s resolution data were postintegrated to give 1-min averages for this study. In order to obtain a Quiet Day Curve (QDC) for the magnetometer data, 2-4 days were chosen that were as close to each selected event as possible and that exhibited minimal perturbations. The dataset was then averaged for these quiet days and Fourier transformed. The number of harmonics was limited to 6 and then the inverse transform was taken to give the QDC for the event. The total horizontal magnetic perturbations $\delta H$ were obtained from components using $\delta H^{2}=\delta X^{2}+\delta Y^{2}$.

The events in this study were selected based on the availability of the tri-static ion drift velocity data from EISCAT.

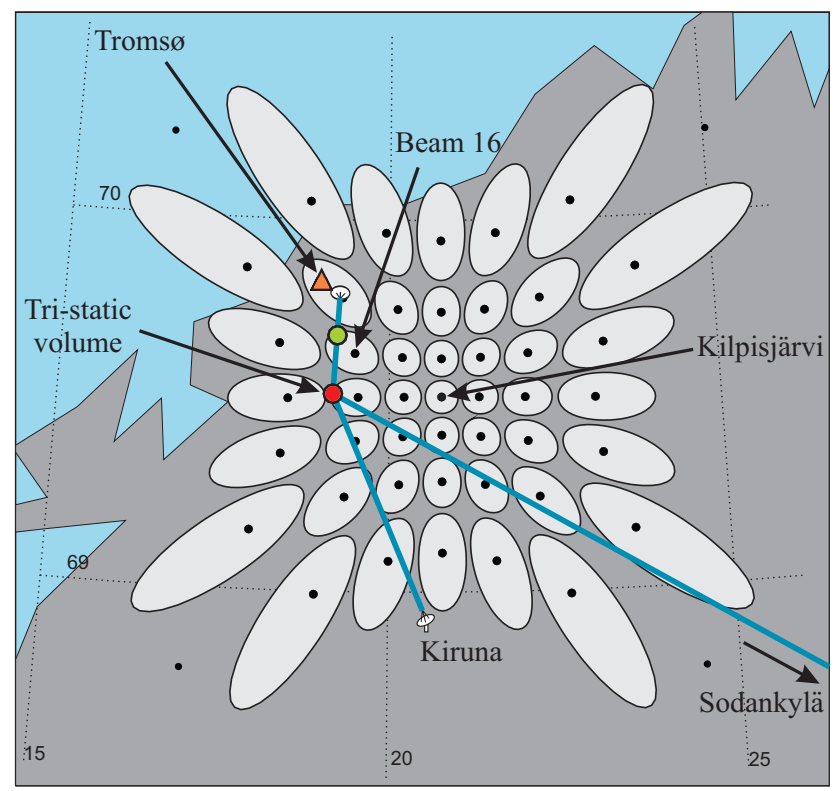

Fig. 1. Experimental setup diagram showing the IRIS and EISCAT fields-of-view. The dots (ellipses) represent the intersection of the principal directions $(-3 \mathrm{~dB}$ riometer beams) with the ionosphere at a height of $90 \mathrm{~km}$. The $-3 \mathrm{~dB}$ beams at the corners of the array are not shown as the antenna side lobes are too large. The 3 blue lines show the receiver beams from the EISCAT in the CP1-K common mode. The red circle shows the position of the tri-static volume at an altitude of $278 \mathrm{~km}$. The green circle illustrates the position of the tri-static volume projected down the magnetic field line to $110 \mathrm{~km}$ in the E-region. At this height it is closest to beam 16 of the IRIS. The orange triangle is the position of the Troms $\varnothing$ magnetometer, which maps close to the projected tri-static volume.

The data from the CP1-K common mode for 46 days in 1995-1999 were analysed. An extended dataset comprising all available IRIS and IMAGE Troms $\varnothing$ data in February 1995-January 1999 was also used in one part of this study (Sect. 3.3). All EISCAT velocity measurements above $2000 \mathrm{~m} / \mathrm{s}$ and all IRIS absorption measurements below $0 \mathrm{~dB}$ were excluded. The effects of radio scintillations due to the passage of Cassiopeia over the IRIS field-of-view (FoV) were also removed from the IRIS data. The data from IRIS and IMAGE were then temporally matched with the EISCAT data to form a dataset comprised of simultaneous measurements.

The EISCAT conductivities were calculated from the electron densities and ion and electron temperatures measured by EISCAT using a method described by Makarevitch et al. (2004). The height-integrated conductivities or conductances were then computed using the height range of 90-275 km. Similar conductance estimates were employed by numerous previous studies (e.g. Davies and Lester, 1999; Senior et al., 2007). To determine the particle component of conductances, the solar effects were removed from each conductance 

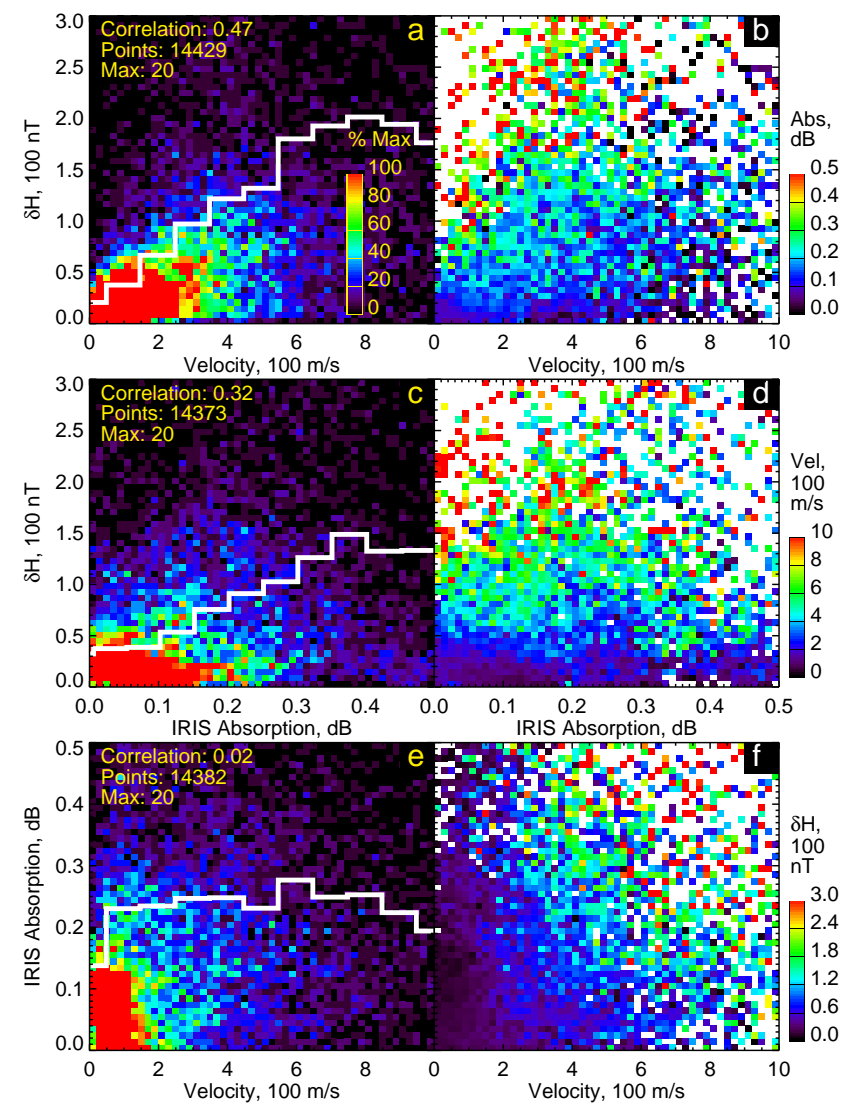

Fig. 2. 2-D plots of $(\mathbf{a}, \mathbf{b})$ the IMAGE magnetic perturbation $\delta H$ versus the EISCAT ion velocity, $(\mathbf{c}, \mathbf{d})$ the IMAGE magnetic perturbation versus the IRIS absorption, and (e, f) the IRIS absorption versus the EISCAT ion velocity for the entire dataset. In panels (a), (c) and (e) the occurrence of points for each cell up to a maximum of 20 is shown, colour-coded in percentage of maximum as indicated by the colour bar in panel (a). In panels (b), (d) and (f), the cells are colour-coded in the mean absorption, velocity, and perturbation, respectively, as shown on the right of each panel. The correlation for the entire dataset is shown in the top-left corner of panels (a), (c), and (e) along with the total number of points. The bin-averaged parameter dependence is shown by the white histogram.

(Hall or Pedersen) using a square root of squares technique (Brekke and Hall, 1988): $\Sigma_{\text {Part }}^{2}=\Sigma_{\text {Tot }}^{2}-\Sigma_{S}^{2}$, where $\Sigma_{\text {Part }}$ and $\Sigma_{S}$ are the particle and solar components of conductance, respectively, and $\Sigma_{\text {Tot }}$ is the total conductance. The solar component was calculated from the solar conductance model proposed by Robinson and Vondrak (1984).

\section{Observations}

\subsection{Point-by-point comparisons and correlation analysis}

In this section the relationships between magnetic perturbations, CNA, and the field-perpendicular component of the ion drift velocity are explored for both the entire dataset and for different MLT sectors. The data have been matched spatially and temporally, comprising of data points with three values representing the electrojet current strength, the Hall conductance, and the ionospheric electric field, respectively. Two parameters are plotted against each other in a 2-D format while either the occurrence or the mean value for the third parameter is represented by the colour of the plot cell.

The comparisons over the entire dataset are displayed in Fig. 2. Panels $(a, b)$ to $(e, f)$ display the 2-D plots of perturbation versus velocity, perturbation versus absorption, and absorption versus velocity, respectively. The left-column panels show the percentage occurrence of points for each cell, while the right-column panels are colour-coded in absorption, velocity, and perturbation, respectively, with the colour showing the averaged value over all measurements inside a given plot cell. The white histogram is the bin-averaged parameter variation. The linear Pearson correlation co-efficient for the entire dataset is shown in the top-left corner of leftcolumn panels along with the total number of points. The panels from $(\mathrm{a}, \mathrm{b})$ to $(\mathrm{e}, \mathrm{f})$ contain $91 \%, 83 \%$ and $81 \%$, respectively, of the total points available after the previously defined restrictions were applied (Sect. 2).

Figure 2a shows an overall increase of perturbation with ion velocity, which is reflected in the overall positive correlation co-efficient of 0.47 and in the steady increase of the bin-averaged perturbation with ion velocity. There is some structuring in the corresponding panel (b) due to absorption variation which is evident in the colour coding. That is, the red and yellow cells tend to be in the top-left part of the diagram with steady increase occurring in mean absorption as $\delta H$ increases and velocity decreases. This suggests that conductance also contributes to the electrojet current.

Another way of studying the perturbation-absorption (current-conductance) relationship is presented in panels $(\mathrm{c}, \mathrm{d})$ where a direct comparison is made between perturbation and absorption; the colour coding in panel (d) is now in the EISCAT ion velocity. This panel reveals some increase in magnetic perturbation with absorption. However, the correlation co-efficient is somewhat lower in panel (d) (0.32 versus 0.47 ). The colour coding in velocity, on the other hand, displays obvious structuring indicating an increase in perturbation with velocity (red cells are higher and blue cells are lower), in agreement with the correlation between perturbation and ion velocity observed in panel (b). The analysis of the entire dataset thus suggests that, on average, the electric field control of current is stronger than that of conductance 


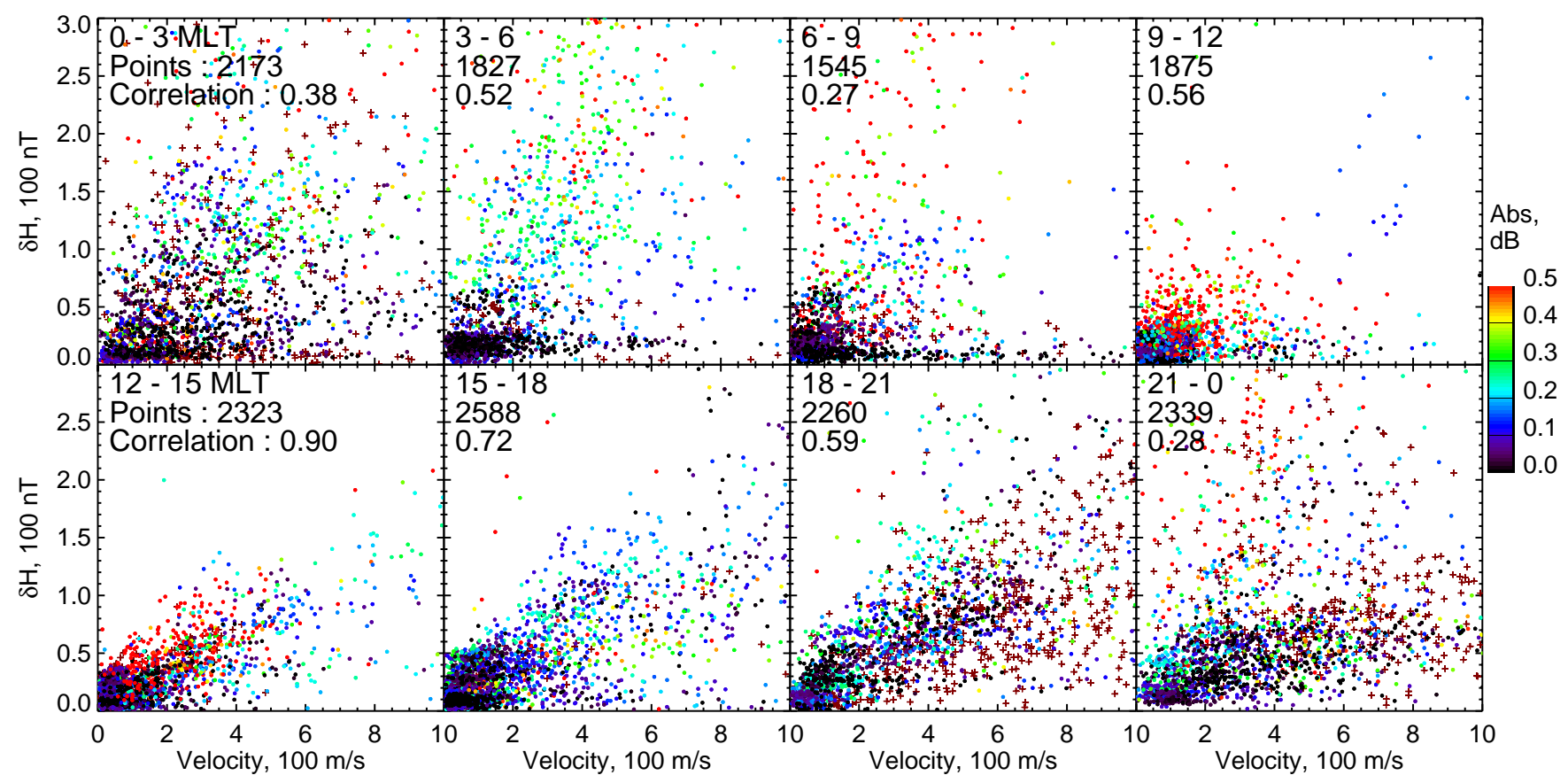

Fig. 3. Scatter plots of magnetic perturbation versus ion velocity for different MLT sectors. The correlation and total number of points are also shown in the top-left corner of each panel. The colour coding is in absorption as shown to the right of the plot. The points with no simultaneous absorption measurements are shown by dark red pluses. The bin-averaged perturbation is shown by the red histogram.

as the correlation was higher in panel (b) and structuring was more pronounced in panel $(\mathrm{d})$.

The comparisons between perturbation and ion velocity (absorption) have demonstrated positive correlation and obvious structuring in Fig. 2b (Fig. 2d). In panels (e, f) absorption and velocity are compared directly with the colour coding in panel (f) in the IMAGE magnetic perturbation. This comparison reveals no significant correlation or anticorrelation between these parameters for the entire dataset. Data does seem, however, to be spread along the both axes in panel (f) suggesting a possible inverse proportionality relationship, which is also evident in the 2-D occurrence plot of panel (e). This is emphasized in panel (f) by structuring of the cells according to colour with blue (red) cells generally closer to (farther from) the axes. Red cells (large mean perturbations) are observed in the top-right quadrant of the diagram where both absorption and velocity are large.

Although some correlation was found when analysing the three parameters over the entire dataset, the temporal variation of this correlation was not investigated. In the latter part of this section we investigate the time variation of these correlations in three-hour MLT blocks (MLT=UT+2.7). This should give an indication of how the conductance and electric field control of electric current varies with MLT.

In Fig. 3 the data have been divided into 8 three-hour time sectors by MLT. For each time sector the perturbation is plotted against the ion drift velocity. The correlation is shown, along with the number of points in the top-left corner of each panel. The colour coding is in absorption and is the same for all time sectors as displayed on the right of the figure. The dark red pluses represent points without colour coding information that were nevertheless included in the correlation analysis. The figure shows that the correlation is highest just after magnetic noon (0.90 at 12:00-15:00 MLT) and stays quite high until 21:00 MLT where it sharply decreases to 0.28 . It is minimised in the midnight sector before rising again in the morning sector (03:00-06:00 MLT). The two features of interest in the figure are the rapid increase in correlation at 12:00 MLT and the sharp decrease at 21:00 MLT with the colour coding in absorption also showing some interesting results. There is more data structuring near midnight (where red points are at the top and black points are at the bottom) and less in the afternoon, possibly indicating a higher correlation between absorption and perturbation near midnight and less correlation near noon, which is explored next.

The IMAGE perturbation and the IRIS absorption are compared in Fig. 4, which has the same format as Fig. 3 with colour coding now in the EISCAT ion velocity. The results clearly display the expected pattern: the data show a temporal variation in correlation that is roughly opposite to that observed in Fig. 3. Correlation is highest (lowest) in the 21:00-06:00 MLT (09:00-21:00 MLT) sector. In other words, it is maximised in the post-midnight sector and minimised in the afternoon sector. There is also a sharp increase in correlation across the 21:00 MLT border and a moderate decrease through the 03:00-09:00 MLT sector. 


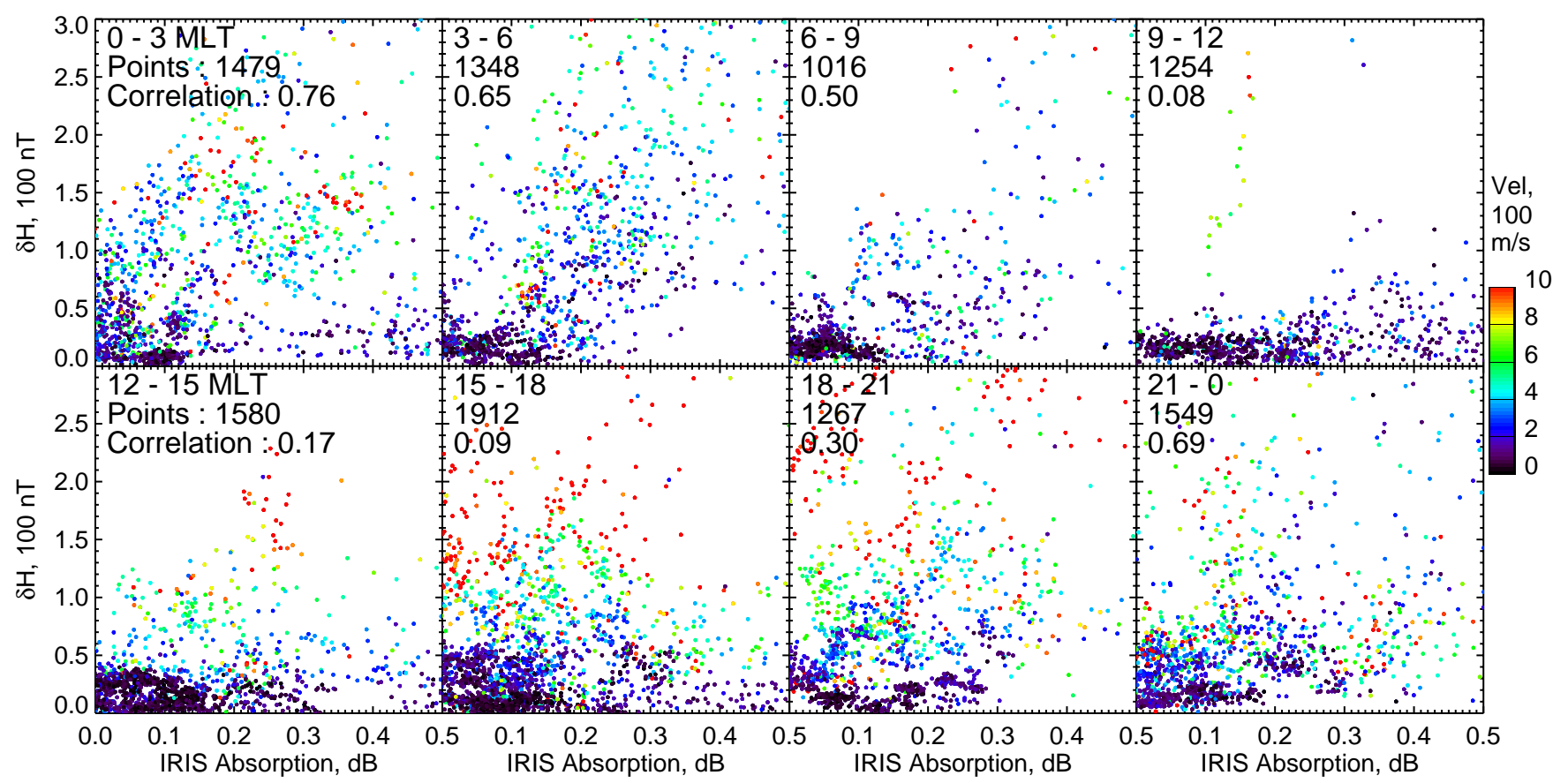

Fig. 4. The same as Fig. 3 except magnetic perturbation and absorption are compared and colour-coded in velocity.

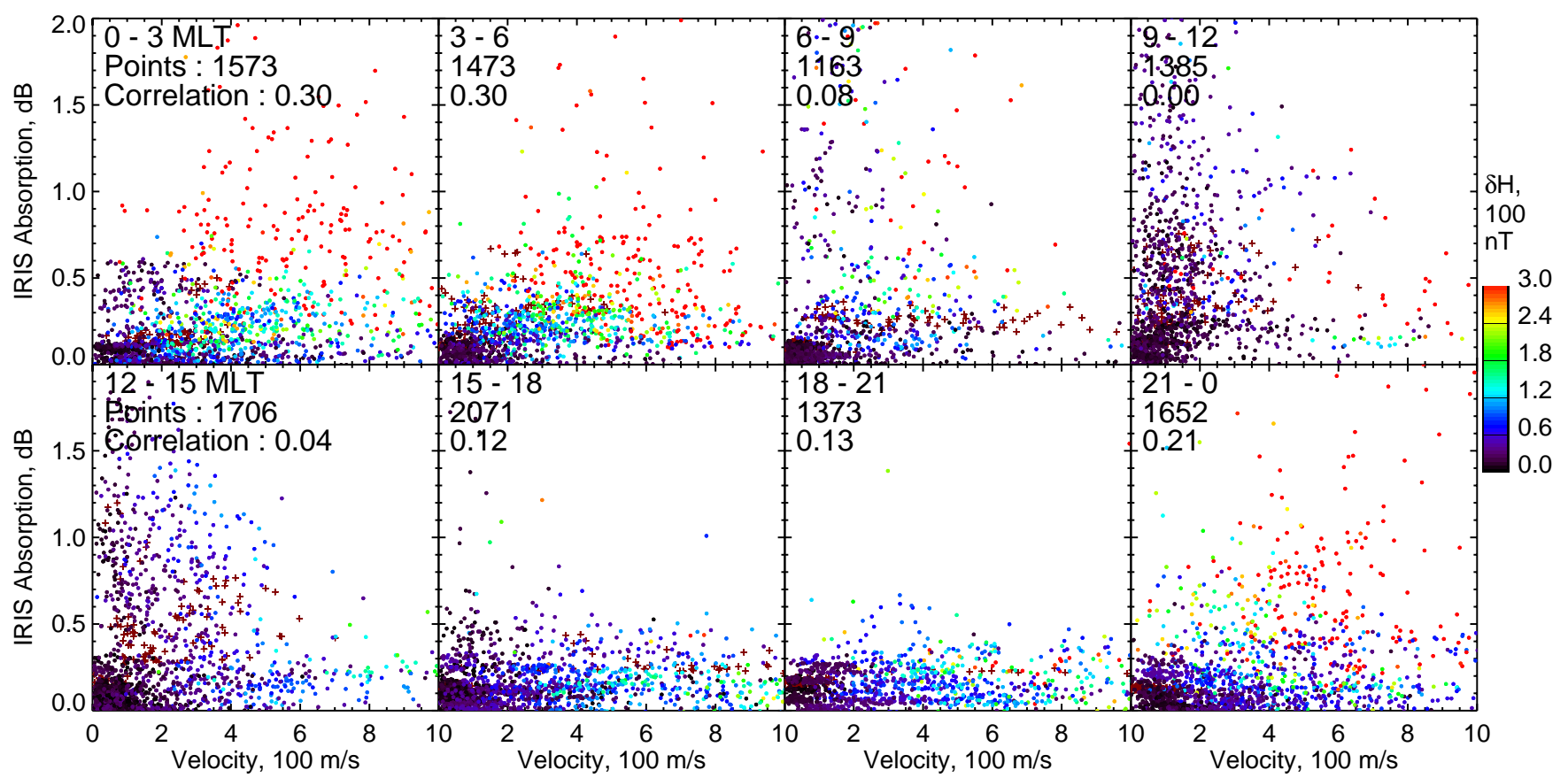

Fig. 5. Same as Fig. 3 except absorption and velocity are compared and colour-coded in perturbation.

To complete the analysis, the relationship between absorption and ion velocity is examined in Fig. 5, where points are now colour-coded in perturbation. The correlation is highest in the post-midnight/early-morning sector 00:00-06:00 MLT (0.30) and very low for the other time sectors. There is, however, some structuring in the 09:00-12:00 and 12:0015:00 MLT sectors where points tend to be close to both axes. This suggests an inverse proportionality between the two parameters in these time sectors similar to that observed in Figs. 2c, d. This feature is further investigated in Sect. 3.5. 

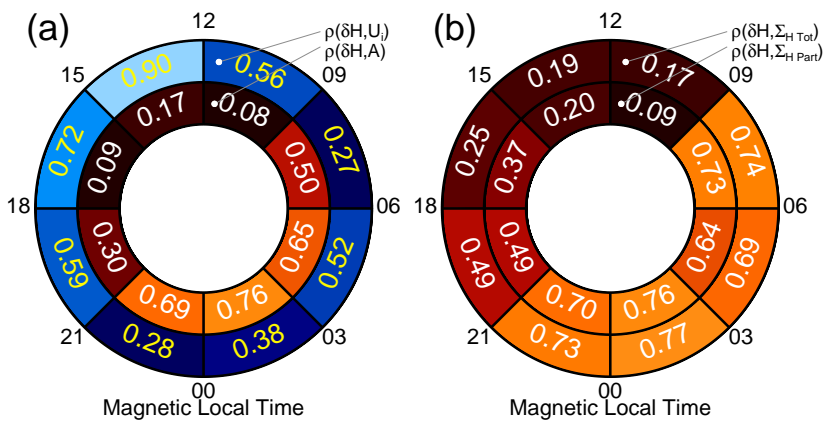

Fig. 6. Clock diagrams showing the correlation of magnetic perturbation (a) with ion velocity (absorption) in blue (red) and (b) with the total (particle component) Hall conductance in outer (inner) circular band for different MLT. The colour coding is representative of the correlation from low (dark) to high (light).

\subsection{Correlation clock diagrams}

The results of the correlation analysis presented above are summarised in Fig. 6a which presents the level of correlation for each 3-h time sector both numerically and by the colour of the sector with lighter (darker) shades representing a higher (lower) correlation. The correlation co-efficients between magnetic perturbations and ion velocity (absorption) $\rho\left(\delta H, U_{i}\right)[\rho(\delta H, A)]$ are indicated in blue (red) colour in the outer (inner) circle. Figure 6a suggests that, overall, the electric field exerted more dominance over the electrojet currents than the conductance. Thus both the overall and maximum correlations are higher for $\rho\left(\delta H, U_{i}\right)$ than $\rho(\delta H, A)$ : 0.47 and 0.90 versus 0.32 and 0.76 . Also, roughly complementary trends/relationships are observed in Fig. 6a, i.e. the current-velocity correlation is substantial whenever the current-absorption correlation is low and vice versa.

We have also performed a similar correlation analysis with the actual Hall conductance rather than with its proxy. The magnetic perturbation correlations with the total (with solar contribution) and particle component of Hall conductance were determined for each 3-h MLT sector. The results are presented in Fig. 6b. All three types of correlation shown in red in Fig. 6 show a very similar picture. Recalling that CNA is not representative of the Hall conductance at 15:00-19:00 MLT (Senior et al., 2007), we observe that the $\rho(\delta H, A)$ and $\rho\left(\delta H, \Sigma_{\text {Part }}\right)$ correlations exhibit a generally good agreement outside of the 15:00-21:00 MLT sector. The $\rho\left(\delta H, \Sigma_{\text {Tot }}\right)$ and $\rho\left(\delta H, \Sigma_{\text {Part }}\right)$ are also quite close with the differences most pronounced on the dayside, in particular at 09:00-12:00 and 15:00-18:00 MLT. The only observation inconsistent with our simple paradigm suggesting that absorption is a good proxy for particle component of conductance is at 06:00-09:00 MLT when $\rho(\delta H, A)<\rho\left(\delta H, \Sigma_{\text {Part }}\right) \cong \rho\left(\delta H, \Sigma_{\text {Tot }}\right)$, which may be due to a failure of the solar conductivity model to fully remove solar effects for this particular subset of data.

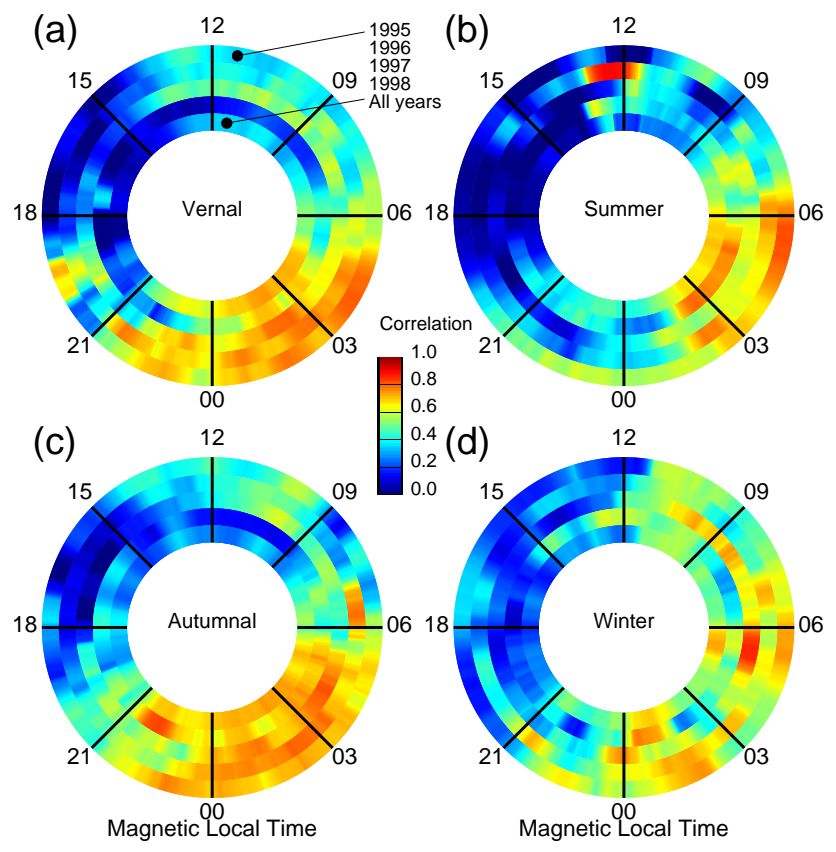

Fig. 7. Clock diagrams showing the correlation co-efficients between magnetic perturbations and absorption for 1-h boxcar correlations calculated at 1-min resolution for different seasons. The colour coding is representative of the correlation as indicated by the colour bar. The circular bands in each panel represent different years as indicated at the top of the diagram.

\subsection{Auroral absorption and currents for different seasons}

In our previous analysis, the dataset was limited to 46 days with simultaneous EISCAT ion velocity data. Our novel approach involving simultaneous and coincident magnetometer and riometer measurements provides a unique opportunity to explore in more detail the statistical current-conductance relationship (using CNA as a proxy). In this section, we employ an extended Troms $\varnothing$ magnetometer/IRIS riometer dataset collected over the 4 years February 1995-January 1999. This period is selected to include 81 days around equinoxes and solstices, that is why 4 calendar years 19951998 are shifted by 1 month. Both datasets have 1-min time resolution so that up to 1440 data points are available for each day. The large amount of data also allowed us to improve drastically the time resolution of the correlation analysis. This has been done by calculating the boxcar correlation co-efficients for a 1-h period centered on every minute. The boxcar correlation dataset thus comprised 1440 values (for each min) with each of them calculated using up to $60 N_{d}$ points, where $N_{d}$ is the number of days in a considered period.

Figure 7 includes 4 clock diagrams, similar to Fig. 6, containing data restricted to 81 days centered on the equinoxes and solstices with panels (a) to (d) showing correlations for 


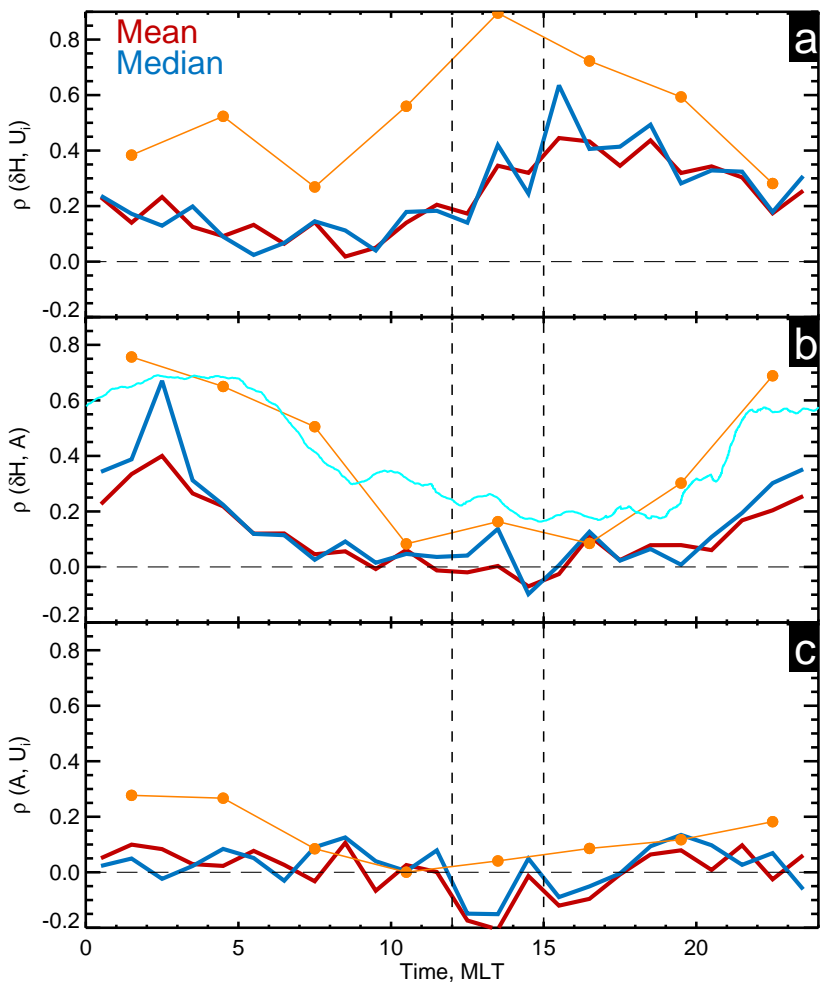

Fig. 8. Line plots of the hourly correlation co-efficients between (a) perturbation and velocity, (b) perturbation and absorption, and (c) absorption and velocity. The red (dark blue) line represents the mean (median) value of the hourly correlation co-efficients. The orange dots and lines represent the 3-hourly correlation coefficients from Figs. 3-5. In panel (b) the light blue line shows a 1-h boxcar correlation computed using an extended dataset.

spring, summer, autumnal and winter periods, respectively. In addition, the data has been further restricted to specific years as shown by different circular bands $\left(N_{d}=81\right)$. The year specified for each circular band in panel (a) is that at the start of each 81-day period. The correlation co-efficients for all years combined are also shown in the innermost circular band $\left(N_{d}=324\right)$. The correlation is represented by the colour as shown by the colour bar in the centre of the plot.

Figure 7 shows a lot more detail in correlation as a function of MLT than Fig. 6. For majority of the years, the region of high correlation extends from 21:00 to 06:00 MLT during the equinoctial periods, panels (a) and (c), which is also reflected in all-years correlations. During the summer period, panel (b), it is shifted later into the morning and covers a smaller MLT range (02:00-07:00 MLT). There is also an "anomalous" region of very high correlation near local noon for summer 1996. The winter period, panel (d), displays highly variable correlation with no clearly defined region of high correlation consistent over the years. The correlation on the dayside before 12:00 MLT, however, is noticeably higher than in other seasons. One can conclude that the current-absorption correlation exhibits a distinct seasonal dependence. One should bear in mind though that the correlation values at 15:00-19:00 MLT are not representative of the current-conductance correlation (Senior et al., 2007).

\subsection{Hourly correlation analysis}

Figures 3-5 summarised in Fig. 6a revealed that the correlation between the 3 parameters varied throughout the day. The correlation co-efficients were calculated for each 3-h sector. In Sect. 3.3 the correlation co-efficients were also computed using 1-h boxcar periods. In both analyses the correlations were calculated for all events combined together. In this section the correlation is further examined using a different analysis. The correlation co-efficients are calculated at hourly intervals for each event and then averaged over the 46 events for each hourly period in MLT. We will refer to these values as "hourly" correlations to distinguish from " $1-\mathrm{h}$ " boxcar correlations from Sect. 3.3 and "3-h" correlations from Sects. 3.1-3.2. A similar hourly correlation analysis was employed earlier by Makarevitch and Honary (2005).

The correlation co-efficient for each hourly period was only calculated and entered into the dataset if there were sufficient number of points in the hourly period. This was determined by comparing the data available for each hour to the maximum number of data points that the instrument was capable of collecting during the hour (typically 30 points for EISCAT and 60 points for IRIS and IMAGE). If this number was greater than $60 \%$ then the number of points was deemed sufficient.

In order to compare the results from the hourly correlation analysis with those presented in Figs. 3-6, the mean and median hourly correlation co-efficients were calculated over the entire dataset for each of the 3 pairs of parameters. These are presented as a function of MLT in Fig. 8. The 3-h values from Figs. 3-5 are also shown for reference by the orange lines. The extended IMAGE/IRIS dataset of Sect. 3.3 was employed in panel (b) to compute 1-h boxcar correlations (light blue) over the entire 4-year dataset $\left(N_{d}=365.25 \times 4=1461\right)$.

The 1-h boxcar correlations for the extended dataset show very similar variation to 3 -h values. Panel (a) clearly shows the hourly correlation $\rho\left(\delta H, U_{i}\right)$ starting low in the midnight to early morning sector, then rising quickly after 12:00 MLT and reaching a maximum just after the 12:00-15:00 MLT sector. It then drops off more smoothly through the afternoon. An almost opposite pattern is observed between perturbation and absorption $\rho(\delta H, A)$ in panel (b). The correlation is highest just post-midnight between 02:00-03:00 MLT and smoothly drops off to reach a minimum during the 12:00-15:00 MLT sector. It then rises again to higher values pre-midnight. Panel (c) displays a low correlation $\rho\left(A, U_{i}\right)$ throughout the day with minimal variation, although a small anti-correlation is observed in the afternoon. All these results are consistent with those from the previous analysis as both the mean and median trends behave in a similar fashion to the 
3-h correlations. The hourly correlations are smaller than either 3-h or 1-h boxcar values, which is most likely indicative of some over-averaging occurring in this approach.

\subsection{Electron heating absorption}

The two analyses presented in the previous sections (Figs. 3 and 8) revealed that the data from the afternoon sector exhibited enhanced correlation between magnetic perturbation and ion velocity. This suggested that the electric field is a predominant factor in determining the electrojet current strength in the afternoon. One can argue that the correlation between conductance and electric fields should be maximised during the periods when the correlation between perturbation and ion velocity is close to 1 . In this situation, the electric field affects the current both directly (as current is proportional to the electric field $J \propto E$ ) and indirectly through conductance, collision frequency, and temperature $\left(J \propto \Sigma \propto v_{e} \propto T_{e}^{1 / 2}\right)$ with the latter increasing with electric field. This should result in the maximum correlation observed. To test this idea, 9 days exhibiting high hourly correlations between perturbation and ion velocity were extracted from the dataset. On average, the period of maximum correlation for each interval was found to be close to the high correlation period of 12:00-15:00 MLT in Fig. 3 and this 3-h period was selected as the common period for all 9 days. The days selected, in "yyyymmdd" format, were: 19950301, 19950302, 19950329, 19950928, 19960214, 19960619, 19961211, 19970515 and 19990212.

Figure 9 displays a comparison between absorption and ion velocity for the high correlation period days. The diagram format is the same as for each panel in Fig. 5. However, in Fig. 9 the dataset is restricted to the 12:00-15:00 MLT sector for the 9 selected days. An inverse proportionality is apparent in the data, similar to that observed in the 09:0015:00 MLT sectors in Fig. 5. The red line represents a curve of inverse proportionality defined by $A=200 / U_{i}$, where $A$ is the IRIS absorption and $U_{i}$ is magnitude of the EISCAT ion drift velocity. The choice of a constant in this simple model is rather arbitrary; for this study a value of $200 \mathrm{~dB} / \mathrm{m} / \mathrm{s}$ was selected to best represent the structuring of the data, in particular that the upper envelope of the data points follows the inverse proportionality curve. As will be argued later, this feature is consistent with the notion of limiting of the current by the magnetospheric generator. No significant correlation between the ion velocity and absorption is found in this approach though.

The electron heating effects are expected to exhibit a threshold in the ion drift velocity as the two-stream instability (which is responsible for electron heating) is only operational when the drift velocity exceeds the ion acoustic speed. The accepted value for the threshold velocity is $\sim 400 \mathrm{~m} / \mathrm{s}$ (e.g. Fejer and Kelley, 1980, and references therein). However, Fig. 9 showed little evidence of absorption increase with the ion drift velocity (expected in the EHA mechanism) either at small or at large, above-the-threshold ion velocities.

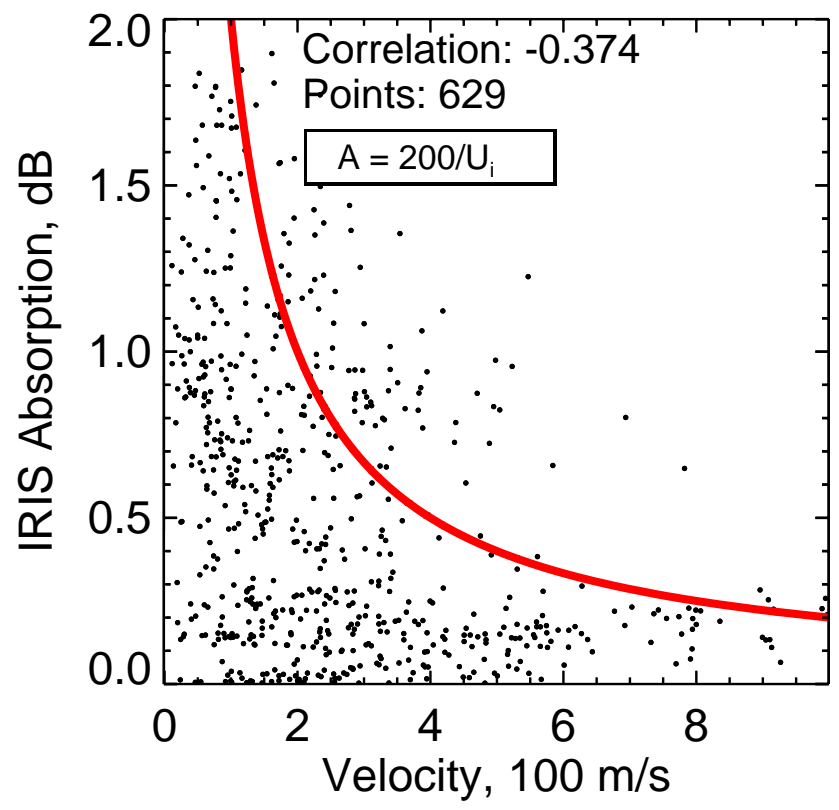

Fig. 9. Scatter plot of absorption versus velocity for the 12:0015:00 MLT time sector for 9 days exhibiting high correlation intervals within this period (see text for details). The format is the same as for each panel in Fig. 5. The red curve represents an inverse proportionality trend described by the equation shown on the graph.

Another approach is to consider the absorption dependence on the electron temperature $T_{e}$ as this is expected to show a more direct effect on absorption through collision frequency: $A \propto v_{e} \propto T_{e}^{1 / 2}$. The electron temperature in the $\mathrm{E}$ region centre, in turn, is expected to show some dependence on the drift velocity above the velocity threshold (e.g. Davies and Robinson, 1997). Consequently, in the following analysis only the $T_{e}$ data points at a height of $111 \mathrm{~km}$ and above the certain temperature threshold were considered. Similar to Davies and Robinson (1997), the electron temperature data were obtained using the alternating code technique. The electron and ion temperatures were derived from the separation and sharpness of the two peaks in the ion acoustic spectra measured by EISCAT; no equality between them was assumed (Rishbeth and Williams, 1985).

The threshold values for temperature were determined for different 3-h MLT sectors as follows. The electron temperatures were plotted versus the ion drift velocity (not shown here). These plots showed a quadratic-like temperature increase with velocity but with different rates of increase for different MLT. This result was reminiscent of the similar quadratic dependence found to well represent the ion acoustic speed $C_{s}=A+B U_{i}^{2}$ with fitting co-efficients $A$ and $B$ dependent on MLT (e.g. Nielsen and Schlegel, 1985; Makarevich et al., 2007). In this study, we used a similar approach, in which a quadratic function of the form $T_{e}=C+D U_{i}^{2}$ was fitted to the data for each MLT sector. The threshold electron temperature $T_{e}^{*}$ for each MLT sector was found by 


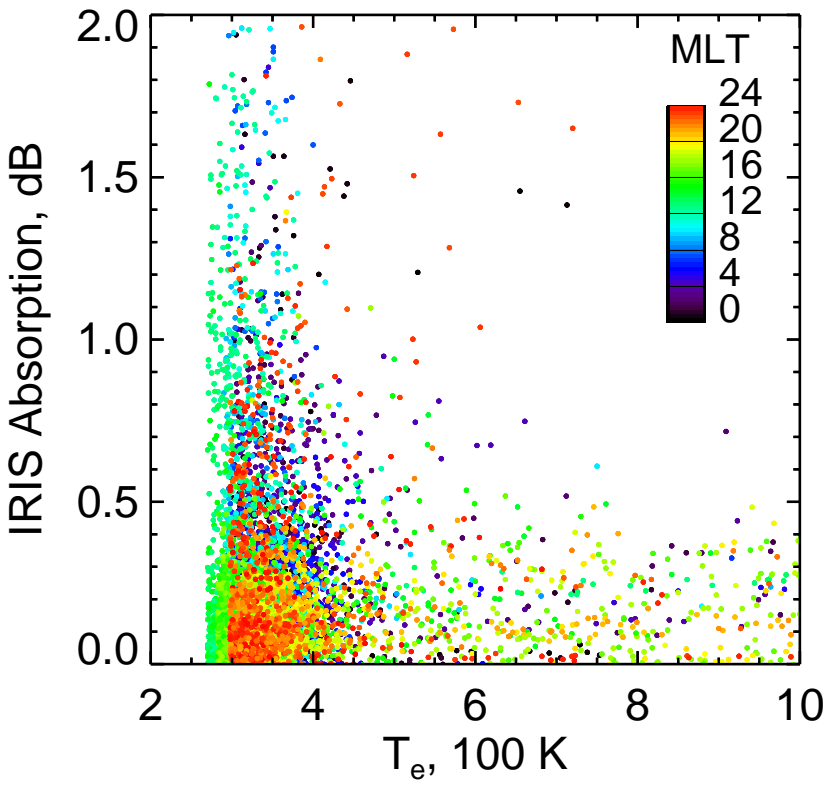

Fig. 10. Scatter plot of absorption versus the electron temperature colour-coded in MLT. The data in each MLT sector is restricted to include temperatures above the threshold electron temperature (see text for details).

substituting $U_{i}^{*}=400 \mathrm{~m} / \mathrm{s}$ into the above equation. Above $T_{e}^{*}$, the electron temperature should be affected by the heating associated with the unstable two-stream waves (see our Sect. 1). Thus one can further explore the electron heating effects by restricting the absorption versus electron temperature data to above-the-threshold values.

The results of this analysis are displayed in Fig. 10 which shows absorption versus the electron temperature colourcoded in MLT. Figure 10 shows that, overall, there appears to be no direct relationship between the two parameters either near the threshold $(300-400 \mathrm{~K})$ or well above it (e.g. $>600 \mathrm{~K}$ ). Interestingly, a possible inverse proportionality relationship can be recognized, similar to that in Fig. 9. One can conclude thus that little evidence of electron heating absorption was observed in this study.

\section{Discussion}

In this paper, the magnetic perturbations, ion velocity, and absorption were compared and the relationship between the current, electric field, and conductance in the auroral region was studied. Compared to previous studies, the MLT coverage was extended to all time sectors including the dayside and the height-integrated characteristic of absorption was used as a proxy for the ionospheric conductance instead of the conductance estimates from incoherent radars. Also, most of the previous statistical studies have drawn their conclusions from analysis and comparison of the averaged/median MLT variations of the electric fields, currents, and conductances. In this study, we used a somewhat different approach in which the measurements were compared and correlated within each MLT sector, Sects. 3.1-3.2. We also employed a significantly larger dataset of simultaneous magnetometer/riometer observations collected over 4 years to statistically investigate the current-absorption relationship in Sect. 3.3. This approach was complemented by the hourly correlation analysis of Sect. 3.4.

\subsection{Electric field and conductance control of currents}

An important thing to bear in mind while interpreting the results of the current study is that riometer measurements provide information on the particle component of conductance. One can expect that on the dayside the correlation between the current intensity and total conductance (due to both solar radiation and precipitation) would be larger. Figure $6 \mathrm{~b}$ supports this assumption with most time sectors showing larger correlation with the total conductance. The fact that in one time sector (15:00-18:00 MLT) the correlation was significantly smaller ( 0.25 vs. 0.37 ) suggests that this simple view is not necessarily always correct.

From above, one should be careful when drawing conclusions in relation to the relative importance of electric field and conductance based on the $\rho(\delta H, A)$ correlation only. However, it is reasonable to expect that the solar contribution would not change the correlation so significantly so that it will exceed the $\rho\left(\delta H, U_{i}\right)$ correlation in the sectors where $\rho(\delta H, A)$ is low and $\rho\left(\delta H, U_{i}\right)$ is high, i.e. at 09:00-21:00 MLT. Again, this is strongly supported by Fig. 6 that shows that the enhanced correlation due to inclusion of the solar conductance $\rho\left(\delta H, \Sigma_{\text {Tot }}\right)$ still does not exceed $\rho\left(\delta H, U_{i}\right)$. By the same token, if $\rho(\delta H, A)$ is substantially greater than $\rho\left(\delta H, U_{i}\right)$ (at 21:00-09;00 MLT) and hence the particle component conductivity dominates over the electric field, this is likely to be the case for the total conductance as well, which agrees well with Fig. 6.

Another possible complication is that magnetometers provide information on the Hall current rather than the total (Hall and Pedersen) current. However, as absorption is closely related to the Hall conductance, the comparisons performed in this study involve the three parameters that are expected to be related via a simple proportionality rule: $J_{H}=\Sigma_{H} U_{i} B$. One has to nevertheless bear in mind that interpreting the observations in terms of the total current may not be so simple in some cases. One important case though when this is possible is when the Hall and Pedersen currents vary synchronously, which happens when they are both controlled by the electric field. That is, one can assume that high $\rho\left(\delta H, U_{i}\right)$ values imply high total-current-electric-field correlation. This means that our results and conclusions from the dayside are unlikely to be affected, which is important as this was the region that was less investigated in the past. In addition, the same approach involving the Hall current estimates from magnetometer measurements has been adopted 
by other researchers (e.g. Kamide and Vickrey, 1983), so that comparisons with their results will provide a straightforward way of verifying our results and conclusions.

Kamide and Vickrey (1983) and Davies and Lester (1999) demonstrated that on the nightside throughout the eastward convection electrojet the electric field is the dominant factor and that the Hall conductance tends to dominate in the westward electrojet. So the transition from the eastward to westward electrojets is commonly believed to coincide with a transition from electric-field- to conductance-driven currents. In our observations, the transition between MLT sectors with high and low $\rho\left(\delta H, U_{i}\right)$ occurred at 21:00 MLT in Fig. 6a, which is consistent with pre-midnight location of transition from the eastward to westward electrojets.

Interestingly, $\rho\left(\delta H, U_{i}\right)$ values temporarily increased again at 03:00-06:00 MLT. This feature may be related to the transition between the conductivity- and electric-fielddominant regions reported by Kamide and Vickrey (1983) to occur in the late morning (near 03:00 MLT) within the westward electrojet. Extending the same argument further towards the dayside, one can propose that a drop in $\rho\left(\delta H, U_{i}\right)$ at 06:00-09:00 MLT is due to relative weakening of the electric field control in this time sector. Statistically, Sugino et al. (2002) showed that for all $K_{p}$ conditions the electric field orientation switched from southward to northward in the late morning sector, just prior to 12:00 MLT, which is normally a characteristic of a change from the westward to eastward convection electrojets. The substantial increase in $\rho\left(\delta H, U_{i}\right)$ across the 12:00 MLT boundary and considerable $\rho\left(\delta H, U_{i}\right)$ correlations at 09:00-21:00 MLT observed in this study thus suggest that the entire eastward convection electrojet is dominated by the electric field including the dayside.

This is contrary to the situation in the westward electrojet that exhibited consistently large values of $\rho(\delta H, A)$ although variable $\rho\left(\delta H, U_{i}\right)$ correlations. The former feature suggests that the westward electrojet is predominantly conductivity-driven. This result disagrees somewhat with that by Kamide and Vickrey (1983) who demonstrated that the westward electrojet in the late morning sector is electricfield-dominant. However, this result is consistent with the statistical study by Davies and Lester (1999) who also did not observe the opposite transition within the westward electrojet. An increase in $\rho\left(\delta H, U_{i}\right)$ observed in our study at 03:00-06:00 MLT was reminiscent of this opposite transition, however the $\rho(\delta H, A)$ value was still quite substantial (0.65) so that, overall, it is difficult to conclude whether the electric-field-dominant region is observed within the westward electrojet on a statistical basis using the EISCATrestricted events. One possible reason is that this feature may only be seen during substorm intervals. However, the results of Fig. 7 based on the extended dataset showed that conductivity-dominant region is 21:00-06:00 MLT near the equinoxes and only 02:00-07:00 MLT in summer. Thus the statistical behaviour near the equinoxes is in agreement with Kamide and Vickrey (1983).
An extension of the conductivity-driven region into the late morning up to 09:00 MLT suggested by the present study is also in rough agreement with the conductivitydominant interval of 20:00-08:00 MLT proposed by Sugino et al. (2002). These authors observed two peaks of conductivity dominance: one near midnight and one in the late morning around 06:00 MLT. This result also agrees well with our observations that showed a significant drop in $\rho\left(\delta H, U_{i}\right)$ at 06:00-09:00 MLT. One should note that the positive difference between $\rho(\delta H, A)$ and $\rho\left(\delta H, U_{i}\right)$ in the 06:0009:00 MLT sector becomes even larger if solar contribution to conductance is taken into account, Fig. $6 \mathrm{~b}$, so that the above conclusion that the entire westward electrojet is conductivity-dominant is not affected.

Figure 6a also shows that high $\rho(\delta H, A)$ correlation starts abruptly at 21:00 MLT, continues through the midnight sector, and stretches out into the morning sector where it slowly declines. We believe that this slow decline may be attributed to the slowly varying absorption (SVA) effect (Stauning, 1996a) as described below. The energetic particle precipitation could easily ionise particles in the upper D- to lower E-regions to which the Hall conductivity and CNA are most sensitive. Satellite observations employed by Collis et al. (1984) showed that the spectrum of drifting electrons hardens with increasing time up to 09:00 MLT, which for the same particle flux would cause a larger detected CNA and correlation with the Hall current. The sharp fall in $\rho(\delta H, A)$ across the 09:00 MLT border is consistent with this idea. The steady decrease in correlation through the morning sector to 09:00 MLT seems to be inconsistent with Collis et al. (1984), as one would expect the correlation to increase with the hardening electron spectrum. However, the decline in correlation with increasing MLT may be related to the eventual depletion of the electron clouds drifting around the Earth eastward towards the dayside. This results in more energetic but less intense precipitation, which would contribute less to the conductance. Local substorms are also more likely to occur at 19:00-04:00 MLT, which would enhance the conductance and hence the current together with the CNA detected by the IRIS at these times. Substorms thus may account, in part, for the larger correlation observed at earlier MLT.

Coming back to the seasonal dependence of currentabsorption correlation, Fig. 7 revealed that the high correlation region was wider in MLT near the equinoxes than in summer. This may be simply due to the larger absorption values during equinoxes (Ranta et al., 1983), which is itself possibly one of the manifestations of the Russell-McPherron effect (Russell and McPherron, 1973). However, in this interpretation the correlations should be larger in winter than in summer as well, which is not seen in Fig. 7. Another interesting feature that is difficult to explain is that the correlations are lower near midnight 00:00 MLT and higher near dawn 06:00 MLT in summer than near equinoxes so that the high-correlation region is observed at 02:00-07:00 MLT. The second observation may be related to an increase in particle 
number flux on the dayside and a corresponding increase in energy flux in summer as compared to winter reported by Liou et al. (2001) (see their plates 3-5), while the first observation appears not to have a direct counterpart in low-energy $(<10 \mathrm{keV})$ particle observations. This suggests that absorption signatures of the high-energy particles, in particular in the context of their relationship with auroral currents, are significantly different from their low-energy counterparts.

Our observations suggest that, on average, the conductance- (electric-field-) dominant region is centered at 00:00-03:00 (12:00-15:00) MLT. This result is consistent with previous statistical studies that found that the westward electrojet centre was generally located post-midnight (Kamide, 1991). The post-midnight shift was explained by the DP2 (DP1) current system contributing to the westward electrojet at 00:00-06:00 MLT (21:00-03:00 MLT) so that averaging over all events shifts the westward electrojet center, and hence the conductivity-dominant region centre, from midnight to the early morning.

In Fig. 6a, a similar shift (towards later MLT) is also observed in the current-velocity correlation which is substantial at 09:00-21:00 MLT. This overall rotation of the electricfield-dominant region clockwise from the noon-midnight meridian could be related to the properties of the plasma convection pattern as discussed below. The high-latitude plasma convection pattern is known to depend strongly on the direction and magnitude of the IMF $B_{y}$ component (e.g. Heelis, 1984). Statistically, Ruohoniemi and Greenwald (2005) and, more recently, Haaland et al. (2007) demonstrated the lack of mirror-symmetry between the effects of positive and negative IMF $B_{y}$. Similar results for several events during small $B_{y}$ conditions were also reported by Kustov et al. (1998). The significant shift towards earlier MLT in location of the "throat" region (where plasma flows into the polar cap) and the resulting rotation in the convection cells only occurred for positive (negative) IMF $B_{y}$ in the Northern (Southern) Hemisphere (Haaland et al., 2007). This implies that, on average, the transition between the westward to eastward convection electrojet occurs in the late morning sector. The results of the current study are consistent with these findings, as in our observations the transition from the conductance- to electricfield-dominant region also occurred in the late morning, at 09:00 MLT in Fig. 6a.

\subsection{Auroral absorption and electric field}

In Figs. 2e and $2 \mathrm{f}$ absorption was compared with the ion velocity over the entire dataset and a negligible correlation found. Although one expects no direct relationship between absorption (conductance) and plasma convection speed (electric field), various indirect links have been proposed in the past including the electron heating mechanism in which absorption may exhibit an increase with the electric field (see Sect. 1). No such increase was seen in Figs. 2e, 2f, 5,9 . Thus these results indicate that no evidence of electron heating absorption is observed on a statistical basis in the auroral zone. However, an interesting result was that in Fig. 8c some anticorrelation was observed at 12:00-15:00 MLT and that in Figs. 5 (09:00-15:00 MLT) and 9, an inverse proportionality was present.

The inverse proportionality between absorption and plasma velocity found in this study can be explained if a simple model of the magnetospheric generator is used such as that proposed by Robinson (1984), based on the work done by Reiff et al. (1981). In the magnetospheric generator model, the field-aligned currents are assumed to flow in large sheets that are connected in the ionosphere by Pedersen currents. The field-aligned current integrated across one sheet, $J_{0}$, is fixed, but the magnetospheric convection and the electric field adjust themselves to ionospheric conductance variations. Therefore the Pedersen currents, which close the circuit in the ionosphere, are limited by $J_{0}$, so that $\Sigma_{P} E=J_{P} \leq J_{0}$. The observed inverse proportionality relationship between the conductance and electric field can thus be attributed to this limit on the Pedersen current. If the current is limited then any further increase in the electric field must be associated with a decrease in the Pedersen conductance and vice versa. Later this limitation was described in terms of the magnetospheric current generator (or source) as opposed to the voltage generator in which the electric field does not change much regardless of conductance changes (Fujii and Iijima, 1987).

There are two potential problems with this interpretation though. Firstly, the simple magnetospheric generator model suggested by Robinson (1984) would not hold in perturbed current systems such as that observed in the midnight sector when precipitation is the main contributor to currents and DP1 systems are in action. Secondly, the magnetic perturbations are mostly representative of the Hall currents, while CNA correlates best with the Hall conductance (Senior et al., 2007). However, the time period 12:00-15:00 MLT is away from the midnight sector and provided that the Pedersen and Hall currents varied more or less synchronously, the simple model of Robinson (1984) can be employed as a possible explanation of our results. As mentioned, one can certainly expect the two currents to vary synchronously during the high correlation periods between the current and electric field. The data collected in the 09:00-12:00 and 12:0015:00 MLT sectors in Fig. 5 for the entire dataset also showed some evidence of inverse proportionality, which suggests that the especially high correlation between electric fields and the current may not be necessary. Rather, an electric field control during the late morning and early afternoon in general, could be indicative of the magnetospheric generator effect.

Possible cases of electron heating absorption within the dataset were further investigated by considering the absorption dependence on the EISCAT alternating code electron temperature at $111 \mathrm{~km}$ above certain MLT-dependent threshold value, Fig. 10. Overall, there was no direct relationship found between the two parameters either near the threshold 
or well above it in any MLT sector. However, a possible inverse proportionality relationship could be seen in the data, which was reminiscent of the relationship between the absorption and the electric field.

\section{Summary and conclusions}

The relationship between magnetic perturbations, absorption, and ion drift velocity was studied, for the first time, using a temporal correlation analysis technique for different MLT sectors. This analysis method was augmented by an hourly correlation analysis for individual events which displayed a similar MLT variation of mean and median hourly correlations. In addition, an extended perturbation/absorption dataset was considered with correlations showing a distinct seasonal dependence. This study demonstrates the strong potential of riometers in studies of the current-conductance-E-field relationship, particularly in combination with magnetometers.

The current-electric-field correlation was generally higher than the current-absorption correlation, which suggests that, on average, the electric field is more dominant in the control of the current than conductance. The current-electric-field correlation was found to be higher on the dayside (09:00 21:00 MLT). It was maximised in the 12:00-15:00 MLT sector reaching 0.90 , with a sharp increase across the 12:00 MLT border. The current-absorption correlation was higher on the nightside (21:00-09;00 MLT) reaching its maximum of 0.76 in the post-midnight sector (00:00-03:00 MLT). This suggests that the conductance is more dominant on the nightside.

The transition from an electric-field-dominant region to a conductivity-dominant region in the pre-midnight sector (21:00 MLT) was associated in the past with a transition from eastward to westward electrojet currents. The results from the nightside showing predominantly conductivitydriven current were generally consistent with previous statistical studies, with some evidence of the opposite transition observed in the late morning sector near 03:00 MLT, which was previously observed only for individual events. An extension of the conductivity-dominant region to 09:00 MLT with a gradual decrease in the current-absorption correlation was also observed, which was explained as being in part due to energetic electrons drifting eastward around the Earth and gradually precipitating in the morning sector. The analysis of the extended 4-year dataset discovered a distinct seasonal dependence in the absorption-current relationship. The region of high current-absorption correlation was found to be at 21:00-06:00 MLT near the equinoxes, while in summer it was narrower and rotated towards dawn 02:00-07:00 MLT. The significant asymmetry/rotation of the electric-field-dominant region with respect to the 00:0012:00 MLT meridian found in this study, may be related to the lack of mirror symmetry between the effects of positive and negative IMF $B_{y}$ on the plasma convection pattern.
There was no correlation found between absorption and electric field for the entire dataset. Also, no significant correlation was found for different MLT sectors, during periods of high correlation between electric fields and current, or for drift velocities and electron temperatures above the twostream instability threshold. Thus no substantial evidence of electron heating absorption in the auroral zone was found. However, an inverse proportionality was found between the absorption and electric field during the high current-E-field correlation periods and, generally, in the 09:00-15:00 MLT sector, which was attributed to a limit on the Pedersen current imposed by the magnetospheric generator during these time intervals.

Acknowledgements. This research was supported by the Australian Research Council Discovery grant to R. A. M. (project DP0770366). The Imaging Riometer for Ionospheric Studies (IRIS) is operated by the Department of Communications Systems at Lancaster University (UK) in collaboration with the Sodankylä Geophysical Observatory, and funded by the Science and Technology Facilities Council (STFC). The IMAGE magnetometer data are collected as a Finnish-German-Norwegian-Polish-Russian-Swedish project conducted by the Technical University of Braunschweig and Finnish Meteorological Institute. EISCAT is an International Association supported by Finland (SA), France (CNRS), Germany (MPG), Japan (NIPR), Norway (NFR), Sweden (NFR), and the UK (STFC). The authors thank H. Yamagishi, A. Grocott, and Y. Bogdanova for useful discussions and suggestions.

Topical Editor M. Pinnock thanks S. Buchert and another anonymous referee for their help in evaluating this paper.

\section{References}

Amm, O.: The elementary current method for calculating ionospheric current systems from multi-satellite and ground magnetometer data, J. Geophys. Res., 106, 24843-24855, 2001.

Brekke, A. and Hall, C.: Auroral ionospheric quiet summer time conductances, Ann. Geophys., 6, 361-375, 1988, http://www.ann-geophys.net/6/361/1988/.

Browne, S., Hargreaves, J. K., and Honary, B.: An imaging riometer for ionospheric studies, Electronics and Communication, 7, 209217, 1995.

Collis, P. N., Hargreaves, J. K., and Korth, A.: Auroral radio absorption as an indicator of magnetospheric electrons and of conditions in the disturbed auroral D-region, J. Atmos. Terr. Phys., 46, 21-38, 1984.

Davies, J. A. and Lester, M.: The relationship between electric fields, conductances and currents in the high-latitude ionosphere: a statistical study using EISCAT data, Ann. Geophys., 17, 43-52, 1999, http://www.ann-geophys.net/17/43/1999/.

Davies, J. A. and Robinson, T. R.: Heating of the high-latitude ionospheric plasma by electric fields, Adv. Space Res., 20, 11251128, 1997.

Davies, J. A., Lester, M., Milan, S. E., and Yeoman, T. K.: A comparison of velocity measurements from the CUTLASS Finland radar and the EISCAT UHF system, Ann. Geophys., 17, 892902, 1999, http://www.ann-geophys.net/17/892/1999/. 
Fejer, B. G. and Kelley, M. C.: Ionospheric irregularities, Geophys. Rev., 18, 401-454, 1980.

Fujii, R. and Iijima, T.: Control of the ionospheric conductivities on large-scale Birkeland current intensities under geomagnetic quiet conditions, J. Geophys. Res., 92, 4505-4513, 1987.

Haaland, S. E., Paschmann, G., Förster, M., Quinn, J. M., Torbert, R. B., McIlwain, C. E., Vaith, H., Puhl-Quinn, P. A., and Kletzing, C. A.: High-latitude plasma convection from Cluster EDI measurements: method and IMF-dependence, Ann. Geophys., 25, 239-253, 2007, http://www.ann-geophys.net/25/239/2007/.

Heelis, R. A.: The effects of interplanetary magnetic field orientation on dayside high-latitude ionospheric convection, J. Geophys. Res., 89, 2873-2880, 1984.

Kamide, Y.: The auroral electrojets: Relative importance of ionospheric conductivities and electic fields, pp. 385-399, in: Auroral Physics, edited by: Meng, C.-I., Rycroft, M. J., and Frank, L. A., Cambridge University Press, Cambridge, 1991.

Kamide, Y. and Vickrey, J. F.: Relative contribution of ionospheric conductivity and electric field to the auroral electrojets, J. Geophys. Res., 88, 7989-7996, 1983.

Kustov, A. V., Lyatsky, W. B., and Sofko, G. J.: Super Dual Auroral Radar Network observations of near-noon plasma convection at small interplanetary magnetic field Bz and By, J. Geophys. Res., 104, 4041-4050, 1998.

Liou, K., Newell, P. T., and Meng, C.-I.: Seasonal effects on auroral particle acceleration and precipitation, J. Geophys. Res., 106, 5531-5542, 2001.

Lühr, H., Aylward, A., Bucher, S. C., Pajunpää, A., Pajunpää, K., Holmboe, T., and Zalewski, S. M.: Westward moving dynamic substorm features observed with the IMAGE magnetometer network and other ground-based instruments, Ann. Geophys., 16, 425-440, 1998, http://www.ann-geophys.net/16/425/1998/.

Makarevich, R. A., Koustov, A. V., Senior, A., Uspensky, M., Honary, F., and Dyson, P. L.: Aspect angle dependence of the E-region irregularity velocity at large flow angles, J. Geophys. Res., 112, A11303, doi:10.1029/2007JA012342, 2007.

Makarevitch, R. A. and Honary, F.: Correlation between cosmic noise absorption and VHF coherent echo intensity, Ann. Geophys., 23, 1543-1553, 2005, http://www.ann-geophys.net/23/1543/2005/.

Makarevitch, R. A., Honary, F., McCrea, I. W., and Howells, V. S. C.: Imaging riometer observations of drifting absorption patches in the morning sector, Ann. Geophys., 22, 3461-3478, 2004, http://www.ann-geophys.net/22/3461/2004/.

Marple, S. R. and Honary, F.: A multi-instrument data analysis toolbox, Adv. Polar Upper Atmos. Res., 18, 120-130, 2004.

Meng, C.-I., Rycroft, M. J., and Frank, L. A. (Eds.): Auroral Physics, Cambridge University Press, Cambridge, 1991.

Nielsen, E. and Schlegel, K.: Coherent radar Doppler measurements and their relationship to the ionospheric electron drift velocity, J. Geophys. Res., 90, 3498-3504, 1985.
Ranta, H., Ranta, A., Rosenberg, T. J., and Detrick, D. L.: Autumn and winter anomalies in ionospheric absorption as measured by riometers, J. Atmos. Terr. Phys., 45, 193-202, 1983.

Reiff, P. H., Spiro, R. W., and Hill, T. W.: Dependence of polar cap potential drop on interplanetary parameters, J. Geophys. Res. 86, 7639-7648, 1981.

Rishbeth, H. and Williams, P. J. S.: The EISCAT ionospheric radar: The system and its early results, Q. J. Roy. Astron. Soc., 26, 478512, 1985.

Robinson, R. M.: Kp dependence of auroral zone field-aligned current intensity, J. Geophys. Res., 89, 1743-1748, 1984.

Robinson, R. M. and Vondrak, R. R.: Measurements of E region ionization and conductivity produced by solar illumination at high latitudes, J. Geophys. Res., 89, 3951-3956, 1984.

Ruohoniemi, J. M. and Greenwald, R. A.: Dependencies of high-latitude plasma convection: Consideration of interplanetary magnetic field, seasonal, and universal time factors in statistical patterns, J. Geophys. Res., 110, A09204, doi:10.1029/ 2004JA010815, 2005.

Ruohoniemi, J. M., Shepherd, S. G., and Greenwald, R. A.: The response of the high-latitude ionosphere to IMF variations, J. Atmos. Sol. Terr. Phys., 64, 159-171, 2002.

Russell, C. T. and McPherron, R. L.: Semi-annual variation of geomagnetic activity, J. Geophys. Res., 78, 92-108, 1973.

Schlegel, K. and St.-Maurice, J.-P.: Anomalous heating of the polar E region by unstable plasma waves. 1. Observations, J. Geophys. Res., 86, 1447-1452, 1981.

Senior, A., Kavanagh, A. J., Kosch, M. J., and Honary, F.: Statistical relationships between cosmic radio noise absorption and ionospheric electrical conductances in the auroral zone, J. Geophys. Res., 112, A11301, doi:10.1029/2007JA012519, 2007.

St.-Maurice, J.-P., Schlegel, K., and Banks, P. M.: Anomalous heating of the polar E region by unstable plasma waves. 2. Theory, J Geophys. Res., 86, 1447-1452, 1981.

Stauning, P.: Absorption of cosmic noise in the E-region during electron heating events, Geophys. Res. Lett., 11, 1184-1187, 1984.

Stauning, P.: Investigations of ionospheric radio wave absorption process using imaging riometer techniques, J. Atmos. Terr. Phys., 58, 753-764, 1996a.

Stauning, P.: High-latitude D- and E-region investigations using imaging riometer observations, J. Atmos. Terr. Phys., 58, 765783, 1996b.

Sugino, M., Fujii, R., Nozawa, S., Buchert, S. C., Opgenoorth, H. J., and Brekke, A.: Relative contribution of ionospheric conductivity and electric field to ionospheric current, J. Geophys. Res., 107, 1330, doi:10.1029/2001JA007545, 2002.

Walker, J. K. and Bhatnagar, V. P.: Ionospheric absorption, typical ionization, conductivity, and possible synoptic heating parameters in the upper atmosphere, J. Geophys. Res., 94, 3713-3720, 1989. 